\title{
BMJ Open Long-term effect of smartphone- delivered Interval Walking Training on physical activity in patients with type 2 diabetes: protocol for a parallel group single-blinded randomised controlled trial
}

\author{
Laura Staun Valentiner, ${ }^{1,2}$ Mathias Ried-Larsen, ${ }^{2,3,4}$ Kristian Karstoft, ${ }^{2,4}$ \\ Cecilie Fau Brinkløv, ${ }^{2,4}$ Charlotte Brøns, ${ }^{2,5}$ Rasmus Oestergaard Nielsen, ${ }^{1,2}$ \\ Robin Christensen, ${ }^{6}$ Jens Steen Nielsen, ${ }^{7,8}$ Allan Arthur Vaag, ${ }^{2,3,4}$ \\ Bente Klarlund Pedersen, ${ }^{2,4}$ Henning Langberg ${ }^{1,2}$
}

To cite: Valentiner LS, RiedLarsen M, Karstoft K, et al. Long-term effect of smartphone-delivered Interval Walking Training on physical activity in patients with type 2 diabetes: protocol for a parallel group single-blinded randomised controlled trial. BMJ Open 2017;7:e014036. doi:10.1136/bmjopen-2016014036

- Prepublication history for this paper is available online. To view these files please visit the journal online (http://dx.doi.org/10.1136/ bmjopen-2016-014036).

Received 29 August 2016 Revised 13 February 2017 Accepted 23 February 2017

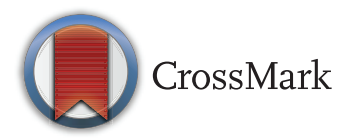

For numbered affiliations see end of article.

Correspondence to Laura Staun Valentiner; lauravalentiner@gmail.com

\section{ABSTRACT}

Introduction: Physical activity is a cornerstone in type 2 diabetes (T2D) rehabilitation. Effective long-term and low-cost strategies to keep these patients' physically active are needed. However, maintaining physical activity behaviour is difficult once formalised interventions end. Structured exercise training supported by mobile technology and remote feedback is potentially an effective strategy. The objective of the trial is to investigate whether mobile health support using the InterWalk application for smartphones is effective in increasing physical activity levels in persons with T2D over time compared with standard care. We investigate whether Interval Walking Training using the InterWalk application is superior to Danish municipality-based rehabilitation in increasing moderate-and-vigorous physical activity levels in patients with T2D across 52 weeks. Secondary, we hypothesise that a motivational programme added from end of intervention to 52 weeks further increases level of physical activity in everyday life in patients with T2D.

Methods and analysis: The trial is a parallelgroup, open-labelled, randomised controlled trial with long-term follow-up at 52 week including patients with T2D. The primary outcome is change in moderate-and-vigorous physical activity. The key secondary outcome includes motivation for physical activity behaviour change. Other secondary outcomes are $\mathrm{VO}_{2}$-peak, strength in the lower extremities. Exclusion criterion is medical contraindication to exercise. We include up to 246 patients and randomly allocate them into a control (standard group) or an experimental group (8-12 weeks of IWT supported by the smartphonebased InterWalk application) in a 1:2 fashion. After intervention, the experimental group is randomly

\section{Strengths and limitations of this study}

- The trial is the largest of its kind, and meets the criteria for high-quality randomised controlled trials with central randomisation and use of valid and reliable measures.

- The trial has a long-term follow-up period of 52 weeks from baseline.

- The trial has high external validity with relevance for the clinical setting as it is performed within clinical practice and includes a limited use of inclusion/exclusion criteria.

- The trial has several collaborators in the set-up, which may cause variation in the testing procedures.

- Lack of clinical end points, for example, depression and mental health.

- The length of the intervention periods varies between the three collaborating municipalities may influence the results of the trial.

- There may be a risk of contamination in the control group as the InterWalk application can be downloaded in App Store.

allocated into two follow-up conditions with unsupervised IWT with or without motivational support until 52-week follow-up. The intention-totreat principle is applied.

Ethics and dissemination: The local regional Research Ethics Committee in Denmark ( $\mathrm{H}-1-2014$ 074) and the Danish Data Protection Agency (j.nr. 2014-54-0897) have approved the trial. Positive, negative or inconclusive results will be disseminated in scientific journals and conferences.

Trial registration number: NCT02341690. 


\section{INTRODUCTION}

Physical activity (PA) is a cornerstone in the clinical care of patients with type 2 diabetes (T2D). ${ }^{1}$ PA has beneficial effect on glycaemic control and other key metabolic risk factors, ${ }^{2}{ }^{3}$ as well as improvements in quality of life. ${ }^{4}{ }^{5}$ Furthermore, supervised long-term PA interventions have proven effective in improving glycaemic control. $^{2}{ }^{6}$ However, experimental evidence does not support the efficiency of advice about PA alone. ${ }^{3}$ As the number of patients with T2D is estimated to rise to 500

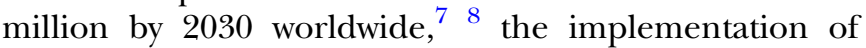
structured, long-term and supervised exercise regimes constitutes a large societal challenge and is not feasible. Thus, novel strategies to increase PA among patients with T2D are needed.

Smartphones have been used as a tool to register exercise, diet, weight and plasma glucose levels, but the evidence for using a smartphone as an exercise device and a self-management tool in the diabetes care is lacking. ${ }^{9}$ However, emerging evidence suggests that eHealth solutions using information and communication technologies $^{10}$ can educate and engage patients with T2D in long-term self-management. ${ }^{11-13}$ Owing to the large ingress and ownership of smartphones, a smartphonesupported approach could prove feasible in increasing PA among patients with T2D and accommodate the increasing prevalence of T2D.

Efficiency of Interval Walking Training (IWT) in patients with T2D has been established. ${ }^{14}$ In the study by Karstoft $e t a l$, IWT was administered and monitored using a small exercise computer (JD-mate). IWT induced significant improvements in physical fitness level, body composition and glycaemic control and in adherence of $>85 \%$ over a 16 -weeks period. ${ }^{14}$ The potential benefits of implementing eHealth solutions in T2D management and the apparent health benefits of and adherence to IWT gave rise to the development of the InterWalk application (InterWalk app) to deliver IWT. ${ }^{15}$

There is a lack of knowledge about the integration of $\mathrm{PA}$ in the everyday life of the patient following rehabilitation interventions. ${ }^{16}$ It is, however, known that successful behavioural change depends on an ongoing maintenance of individual motivation regarding the behaviour and behavioural change itself. $^{17}$ Furthermore, time since onset of diagnosis, unhealthy behaviour and own beliefs about the cause of the problem, together with the number of previous attempts to change and support from partners, peers and health professionals are of relevance. ${ }^{17}$ This underlines the need for new interventions with a direct focus on motivational support and self-control regarding sustaining a newly acquired behavioural change with PA. To better target and structure manageable interventions related to patients with T2D, understanding patients' individual priorities and values is crucial. In this regard, knowledge of individual motivation and self-efficacy to initiate behavioural changes becomes essential to understand. ${ }^{17}$
This paper presents a detailed protocol for the InterWalk randomised controlled trial and is described in accordance with the SPIRIT guidelines (Standard Protocol Items: Recommendations for Interventional Trials). ${ }^{18}$ Results from the trial will follow CONSORT guidelines (CONsolidated Standards Of Reporting Trials) for non-pharmacological interventions. ${ }^{19}$

\section{Trial objective and hypothesis}

The objective of the trial is to investigate whether mobile support using the InterWalk application for smartphones is effective in increasing PA levels in persons with T2D over time compared with a standard care rehabilitation programme.

We investigate the effectiveness of the implementation of IWT using the InterWalk application in the Danish municipality-based rehabilitation programme and study whether the IWT is superior to standard care in increasing moderate-to-vigorous PA 52 after weeks. Furthermore, we expect that a motivational support programme added from end of the intervention to 52 weeks will increase the PA level in patients with T2D and help maintain a physically active everyday life long term.

\section{METHODS AND ANALYSIS \\ Trial design and setting}

The trial is a 52-week parallel-arm, open-labelled, randomised controlled trial. The participants are randomly allocated into two groups, (1) standard care or (2) experimental group with moderate-and-vigorous PA level, doing interval walking facilitated by the InterWalk app. After an 8-12-week intervention period, patients in the experimental group are allocated to do interval walking using the InterWalk app with or without motivational support provided by the health professionals (see figure 1).

The intervention is conducted at the health promotion centres in Danish municipalities and hospitals that consecutively are included as cooperation partners. The municipality of Copenhagen is the first collaborator in the trial. Patients were referred to rehabilitation by their general practitioner (GP). Trained health professionals (physical therapists, nurses and staff with a master's degree) working at the health promotion centres recruit the patients, deliver the interventions and conduct all testing. The overall organisation of healthcare in Denmark is fully tax-financed with universal access to healthcare services. The Danish model is described in detail elsewhere ${ }^{2021}$ and chronic disease care (including T2D) management is in Denmark based in the Chronic Care Model. ${ }^{22}$

Briefly, GPs refer patients diagnosed with T2D to rehabilitation either at an outpatient clinic within a municipality or at the hospital level in Denmark. The setting depends on stage of the disease, presence of complications and comorbidities, for example, severe heart conditions, depression or mental illness. The municipality-based rehabilitation primarily includes non- 


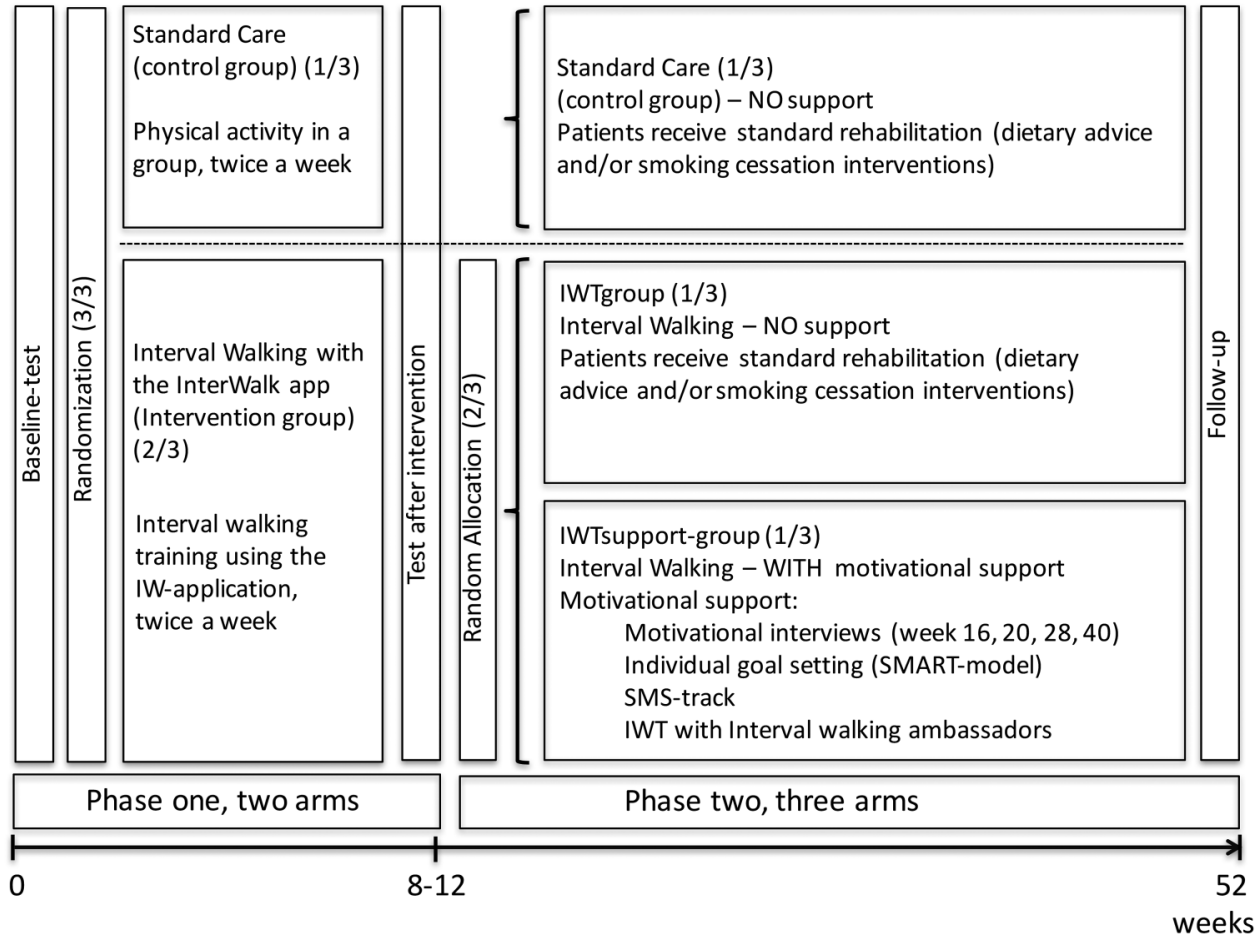

Figure 1 Timeline and overview of interventions in the InterWalk trial. Phase I shows baseline-test, randomisation, intervention and test after intervention. Phase II shows the reallocation of patients in the experimental group who do Interval Walking Training (IWT with the InterWalk app to either IWT with or without motivational support form a health professional). All patients are tested at baseline, after intervention and again at follow-up at 52 weeks.

complicated patients $\left(\sim 80 \%\right.$ of all patients).$^{20}$ If complications or comorbidities are present, patients are referred to treatment at a specialised clinic at a hospital. Patients with a non-complicated course of disease receive rehabilitation consisting of: (1) disease-specific patient education, (2) diet counselling, (3) smoking cessation and (4) exercise. The composition of the individual rehabilitation programme depends, however, on disease progression and on the rehabilitation offered in the municipality-based programme or at the hospital. The general focus is patient empowerment, diseaserelated self-care and prevention of a decay of the functional capacity of patients with T2D.

The InterWalk application is approved by the Danish Data Protection Agency (2008-58-0035). The trial has been registered at http://www.clinicaltrials.org (NCT02341690) on 9 January 2015. Amendments to the protocol will be approved by the Scientific Ethical Committee at the Capital Region of Denmark. Amendments will be reported to http://www.clinicaltrials.org. The trial is conducted in accordance with the Helsinki Declaration.

\section{Participants}

Eligibility

All non-complicated patients diagnosed with T2D (outpatients), referred to a health promotion centre or hospital in the participating municipalities by their GP, are eligible if $>18$ years of age. A flow chart of participants is presented in figure 2 .
The exclusion criteria are medical contraindications to exercise, for example, chronic complications in the locomotive apparatus, painful osteoarthritis or heart conditions. ${ }^{23}$ Information is collected through medical records and at a screening interview with a health professional at the health promotion centre. Furthermore, patients are excluded if they do not want to be physically active in the rehabilitation setting or are already participating in other intervention studies at a health promotion centre. The patients have to be able to talk, read and understand the Danish language (for overview over inclusion and exclusion criterion, see box 1).

If eligible, health professionals give oral and formalised written information with 2 days to consider participation in the trial. Written informed consent is obtained before any additional trial procedures. Enrolment was initiated in January 2015 and recruitment is terminated on 15 December 2016. Last visit of last patient is expected in December 2017.

\section{Interventions}

The trial is developed in collaboration with the health professionals from the municipality of Copenhagen in Denmark. The interventions are designed to reflect the clinical rehabilitation settings in Denmark to increase the likelihood of implementation of the programme following this trial, if proven superior. Health professionals from the municipalities already providing the rehabilitation deliver all interventions in the control and 


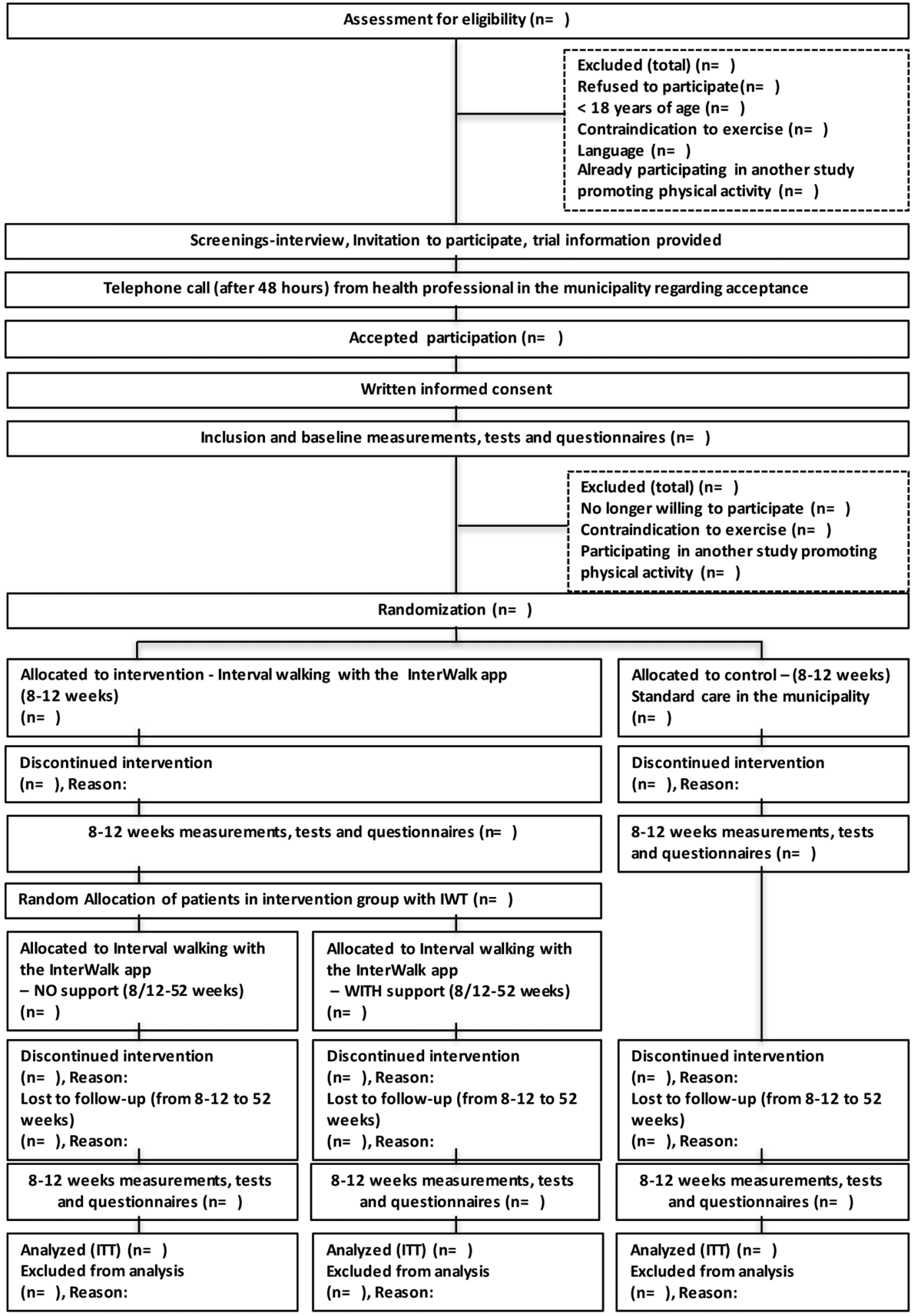

Figure 2 Flow chart, The InterWalk-RCT Trial. RCT, randomised controlled trial.

experimental groups during the trial period. The investigators (LSV, CB and HL) led four workshops (16 hours in total) with the health professionals during year 2014, in which the interventions of the study and work routines were discussed in detail. Furthermore, all participating health professionals underwent a thorough education programme ( 15 hours in total) involving all procedures and manuals in the trial. This was carried out to ensure standardisation of all procedures throughout the trial. In addition, workshops were held from the start of the trial (January 2014) and hereafter every second month. This is carried out to secure that the health professionals follow all procedures and prepared manuals throughout the trial. The applicability of the 


\section{Box 1 Eligibility criteria}

\section{Inclusion criteria}

- Diagnosed with type 2 diabetes

- Referred by general practitioner to a health promoting centre in participating municipality or hospital

Exclusion criteria

- $<18$ years of age

- Medical contraindications to exercise

- Already participating in other exercise trials

- Does not talk and read Danish

trial results is increased here, as the interventions are already part of the rehabilitation programme offered to patients with T2D in the municipalities.

The interventions in the trial consist of two phases (figure 1). In phase I (8-12 weeks), patients are randomised to either a standard care group (control intervention) or an experimental group doing IWT using the InterWalk app. The present trial uses the standard care programme as control intervention and is conducted directly in the clinical setting. All patients receive supervision across phase I. In phase II (40-44 weeks), patients in the standard care group have no follow-up until 52 weeks postbaseline. Patients in the experimental intervention group are in phase II randomly allocated to either IWT with or without motivational support (see section Experimental intervention-IWT with the InterWalk application). Additional rehabilitation care (cointerventions) with disease-specific patient education, diet counselling and smoking cessation is offered to all patients across the intervention groups. The interventions are summarised in table 1.

\section{Experimental intervention-IWT with the InterWalk application (phase I and II)}

Patients allocated to the experimental intervention do interval walking after baseline test provided by health professionals (phase I). IWT is provided by health professionals to smaller groups of 3-12 patients. After the intervention period in phase II, patients are allocated to either receive IWT without motivational support during follow-up (IWTgroup) or IWT with motivational support during follow-up (IWTsupport-group) (figure 1). Trained health professionals conduct the IWT sessions (see below) and the motivational support in phase II. Table 1 gives an example of a typical week with interval walking in the experimental intervention group.

\section{IWT using the InterWalk application}

IWT is performed using the InterWalk app, which works as a personal trainer as well as a monitoring unit allowing researchers to continuously and automatically monitor and store the PA level in a central database (figure 3$).{ }^{15}$

IWT is personalised through a standardised 8 minute walking test, performed with the app before engaging in
IWT. $^{24}$ The InterWalk app guides the user in IWT with repeated cycles of $3 \mathrm{~min}$ fast and $3 \mathrm{~min}$ slow walking. During IWT, the InterWalk app provides the patient with continuous feedback on the walking speed. The patients are able to track exercise history and receive historic feedback on the quality of the IWT session using the app. The feedback system employs the on-board accelerometer of the phone and GPS system to assess intensity and geographical location. Information on training intensity, total number of steps per day, IWT-data and data from the standardised walking test is stored in the smartphone or iPod and automatically transmitted to the central database at the Danish Strategic Research Centre for Type 2 Diabetes (DD2) when connected to Wi-Fi or mobile data network. ${ }^{15}$

\section{Experimental intervention, phase I}

The patient receives a thorough introduction to the InterWalk app with follow-up instructions. The introduction consists of information and test of the app and the patient can ask clarifying questions if needed. A detailed manual regarding the InterWalk app is extradited to each patient. The health professional provides technical guidance and helps the patient to structure an everyday life with IWT during phase I. Patients are encouraged to perform IWT using the InterWalk app (see section: InterWalk Walking Training using the InterWalk Application) as a minimum three times per week, 30-60 min per session, with two of the sessions taking place at the health promotion centre and the third is conducted in the patient's everyday life environment. The aim is that all patients are capable of continuing IWT using the InterWalk app at the end of phase I (table 2).

\section{Experimental intervention, phase II}

In phase II, patients in the experimental intervention group are randomly allocated into two follow-up conditions: with unsupervised IWT without support (IWTgroup) or with motivational support (IWTsupport-group) following the intervention period until 52-week follow-up (see figure 1).

\section{Interval Walking Group without support (IWTgroup)}

All patients in the Interval Walking Group (IWTgroup) are encouraged to continue to perform IWT with the InterWalk app three times a week, 30-60 min per session. The patients are not provided with any follow-up or support from health professionals from the end of the intervention period until follow-up at 52 weeks. However, patients are allowed to contact the health professionals at the health promotion centre at any time if necessary.

\section{Interval Walking Group with support (IWTsupport-group)}

Patients in the Interval Walking Group with support (IWTsupport-group) are also encouraged to do IWT with the InterWalk app three times per week. All patients in the IWTsupport-group receive additional 
Table 1 Interventions in the trial during phase I and II

Phase I Standard care (control intervention)

Group-based training at the health promotion centre Group-based sessions with 4-12 patients.

(control intervention)

- Two sessions per week at the health promotion centre.

- Warm-up exercises.

- Cardiorespiratory exercises.

- Resistance training.

Experimental intervention

- Cool-down period.

Interval Walking Training

- Group-based Interval Walking Training (IWT) using the InterWalk app.

(IWT) $(2 / 3)$

- Introduction to the InterWalk app.

- Follow-up instructions and guidance.

- IWT using the InterWalk (IW) app.

- Three times per week, 60 min per session-twice a week at a health promotion centre in a group and one time alone in everyday life.

- End of phase I: preparation to continue IWT with IW app in the end of the intervention period, through a transition programme.

PHASE II Standard care (control intervention)

Group-based training at the health promotion centre $\quad$ No intervention at the health promotion centre.

(control intervention) (1/3)

- Follow-up at 52 week.

Experimental intervention

IWTgroup (1/3)

IWTsupport-group (1/3)Motivational support

1. Individual motivational interviews with individual goal setting (semi-structured).

2. Short message service (SMS).One weekly SMS and one SMS every forth week.

- No intervention at the health promotion centre.

- Follow-up at 52 weeks.

- Four semistructured individual motivational interviews are scheduled in phase II: week 16, 20, 28, 40.

- Each interview is scheduled to last $30 \mathrm{~min}$

- Individual goal setting related to everyday life following the SMART_-principle.

- The aim of goal setting is to help the patient reflect on their physical activity habits.

- Weekly SMS.

- The reply indicates amount of IWT during the past week (none, 1-2 or 3 or more).

- If no reply for 2 consecutive weeks, or if the reply indicates none walking, then the patient is contacted by phone by a health professional.

- SMS every fourth week.

- Encourages the patient to make a new walking test using the InterWalk app.

- Educated patients with T2D do interval walking in local community near the health promotion centres.

- One session per week

Cointerventions

(across phase I

Additional rehabilitation care (cointerventions) with specific focus areas

Patient education

Disease-related education regarding living with type 2 diabetes, empowerment and self-management and medication handled by either medical doctor, a nurse, physical therapist or dietitian and another patient with type 2 diabetes-group based or individually handled.

Diet counselling

Smoking cessation

The course lasts 10-12 hours and can be either individually or group based.

SMART, specific, measurable, achievable, relevant and timely; T2D, type 2 diabetes. 

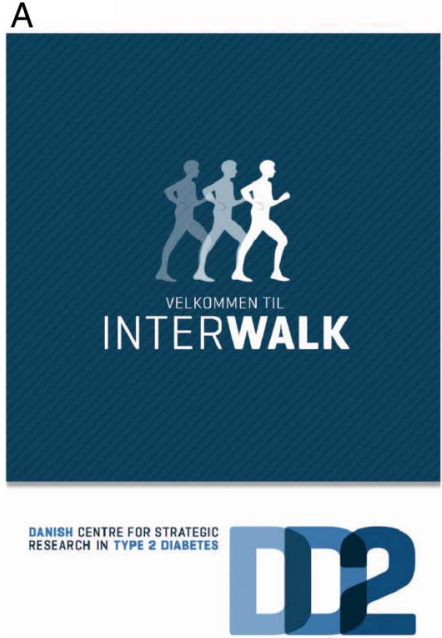
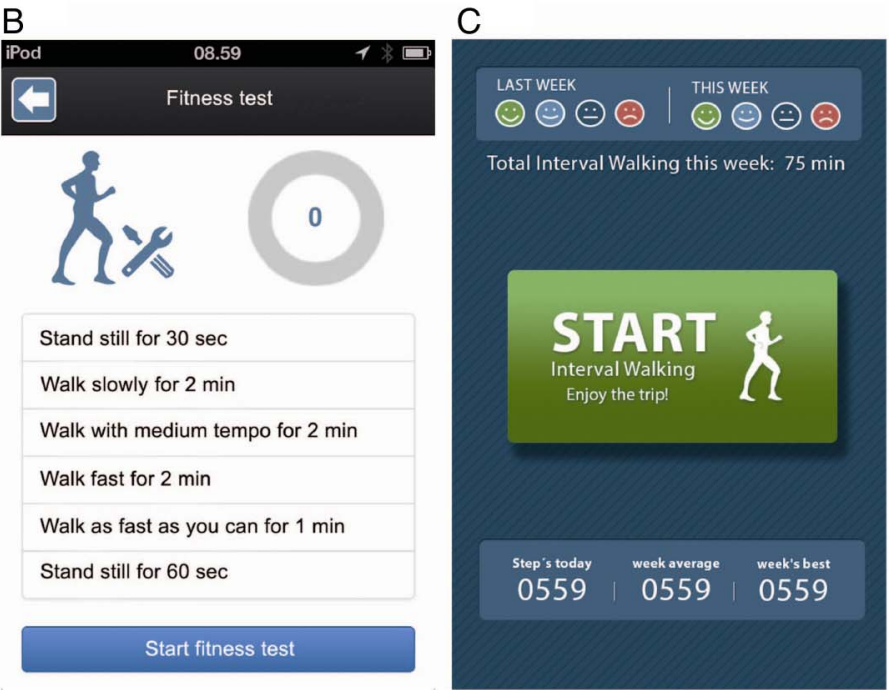

Figure 3 The InterWalk front page (A), protocol for the fitness test in the application (B), the start page for interval walking showing smileys as direct feedback to the user (C) (translated from Danish). ${ }^{16}$

motivational support by the health professionals at the promotion centre (see below for information regarding the motivational support).

\section{Motivational support (phase II)}

The motivational support is summarised in box 2. The motivational intervention consists of four motivational interviews with individual goal setting and weekly short message service via the mobile phone (a SMS-track), (see figures 4 and 5). Furthermore, patients have the opportunity to participate in IWT at predefined timeslots with an Interval Walking Ambassador in the local community near the health promotion centre (see figure 1).

\section{Motivational interview and goal setting}

All health professionals from the municipalities are well educated in conducting the motivational interview with patients. All health professionals working in a health promotion centre in Denmark are obligated to participate in formalised motivational interviewing courses conducted by educators with psychological background. The motivational interview is a semistructured interview used to structure the communication between the patient and the health professional. The interview works as a partnership where focused and detailed questions reveal and visualise the patients motivation and barriers towards behaviour change and acknowledgement of patient autonomy. ${ }^{26}$ Four motivational interviews are scheduled for each patient in the IWT support-group. See box 2 for more detail. The motivational interview seek to help the patients reflect on their physically active habits and to set individual motivating goals related to everyday life. ${ }^{27}$ The health professional secures all notes and reflections from the interview in the InterWalk database. As more than one interview is conducted over the trial period, fidelity may be ensured when the health professional uses the patient's reflections from the former interview to help reflect on motivation and barriers towards changes in the next motivational interview. The goal setting is based on the S.M.A.R.T.-principle derived from the Goal Setting Theory developed by Edwin Locke. ${ }^{25} \mathrm{~S}$ stands for specific (eg, what do I want to accomplish?), $\mathrm{M}$ for measurable (eg, specific and measurable), A for achievable (realistic), $\mathrm{R}$ for relevant (individual goals) and $\mathrm{T}$ for timely (not too big to achieve in a short period). ${ }^{28}$

\section{Short message service (henceforth referred to as text message)}

Enables inexpensive and possibly effective interaction between patients and health professionals at any time or place (Kongstad et al Effectiveness of remote feedback on PA in patients with T2D: a systematic review and meta-analysis of randomised controlled trials. 2016 (submitted)). ${ }^{29}$ Communication as well as feedback is believed to enhance motivation through feelings of competence and relatedness, ${ }^{30}$ which may positively affect adherence to treatment. Text messages have shown positive effect on various health outcomes including diabetes self-management when used in behaviour change interventions. Further, a higher effectiveness and lower attrition rate has been observed when customised and tailored messages is used. ${ }^{29}$ In the present study, the patient receives two types of text messages during phase II (see figures 4 and 5). The first type is a weekly and interactive text message consisting of questions regarding the amount of IWT performed during the past week (see figure 4). The patients are required to give feedback by returning a text message with one of the three response options provided. If the patient does not reply for two consecutive weeks, or if the patient answers that she/he has not been walking, a personal phone call to the patient is conducted from a health professional 


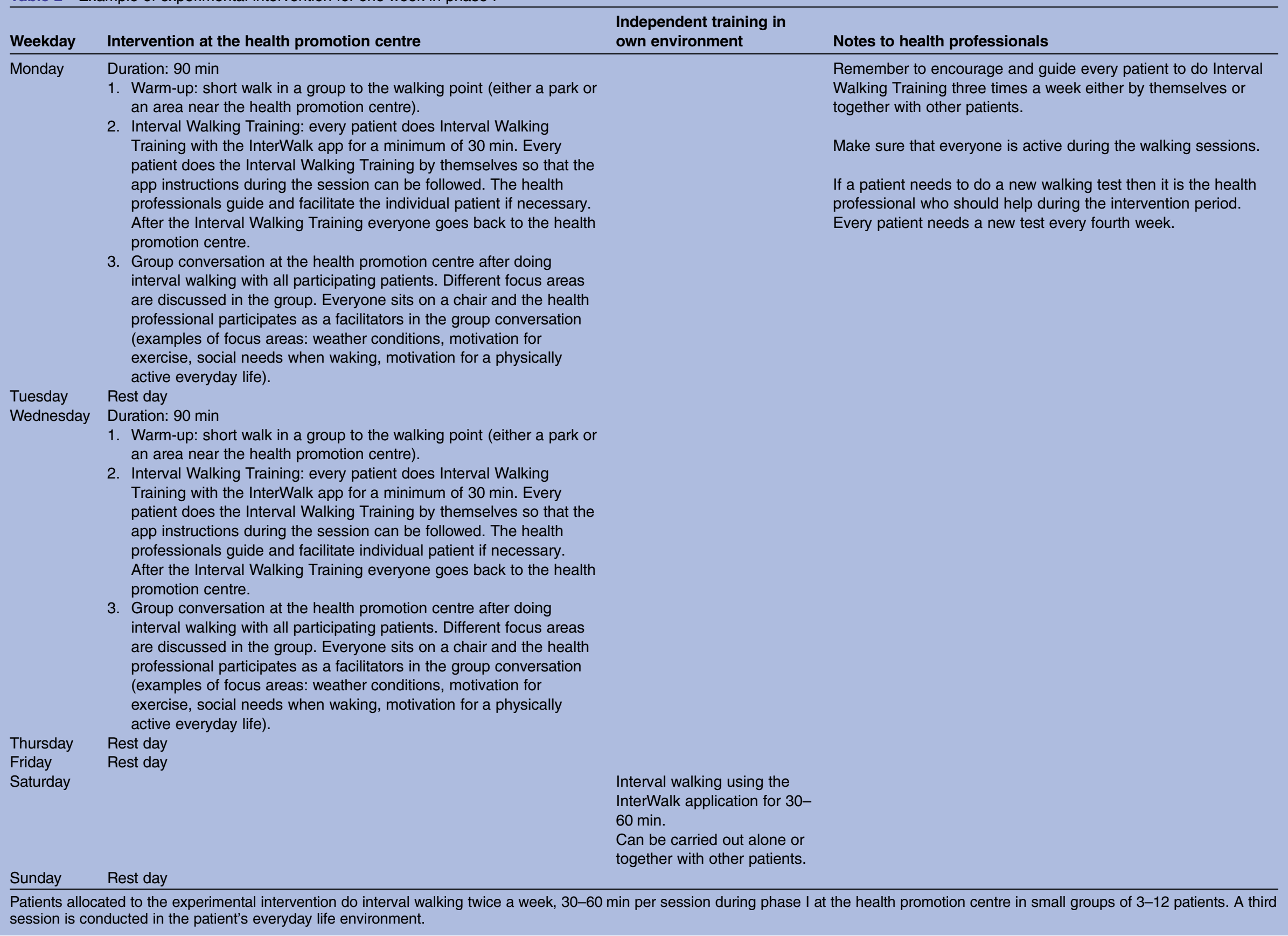

Interval Walking Training: every patient does Interval Walking pp instructions during the session can be followed. The health Walking Training everyone goes back to the heal Group conversation at the health promotion centre after doing professional participates as a facilitators in the group conversation (examples of focus areas: weather conditions, motivation for exercise, social needs when waking, motivation for a physically

Rest day

1. Warm-up: short walk in a group to the walking point (either a park or

(1)

Group conversation at the health promotion centre after doing motivation for exercise, social needs when waking, motivation for a physically

Rest day

session is conducted in the patient's everyday life environment. 
from the local health promotion centre. The aim of the call is to encourage and help the patient to plan IWT to fit the individual life of the patient. The second type of text message is sent every fourth week, encouraging the

\section{Box 2 Motivational interviews with individual goal setting}

\section{Phase II, IWTsupport-group}

Four semi-structured motivational interviews with individual goal setting

- The interviews aim at helping the patient to reflect

- On why and how physical activity can be incorporated in everyday life

- Make informed choices regarding behavioural changes regarding physical activity

- Relate to own everyday life

- Clarify barriers and facilitators of importance for the individual

- Two to three individual goals are set at all interviews

- The goals are based on need in the individual everyday life

A short resume with the individual goal setting is written after all interviews in the database.

The written text is prospectively incorporated in the following interviews with the patient at the health promotion centre The interviews are placed in week 16, 20, 28 and 40 postbaseline test. Goal setting in the interviews is based on the S.M.A.R.T.principle derived from the Goal Setting Theory developed by Edwin Locke. ${ }^{25}$ patients to perform the walking test using the InterWalk app without a requirement of feedback (see figure 5).

\section{Interval Walking Ambassadors}

Using peers to educate other patients with T2D has shown effective in providing ongoing support over a longer period. ${ }^{31}{ }^{32}$ In this study, volunteer patients with T2D are recruited through the Danish Diabetes Association, where a consultant contacts possible patients via email. The Interval Walking Ambassadors do IWT locally in phase II with patients from the IWTsupport group. The Interval Walking Ambassadors receive a 2-day training programme in the participating municipality by researchers from the InterWalk research group. At any time during phase II, patients can attend IWT at predefined timeslots with an Interval Walking Ambassador (see table 1).

\section{Adherence to interventions}

To prevent patients from dropping out during phase I of the trial, patients are contacted by phone by a trained health professional if they fail to appear at the planned training sessions. Up to five attempts are made to contact the patient. In phase II, only patients allocated to the IWTsupport-group are monitored by SMS-track and supervised during the phase until week 52. Patients

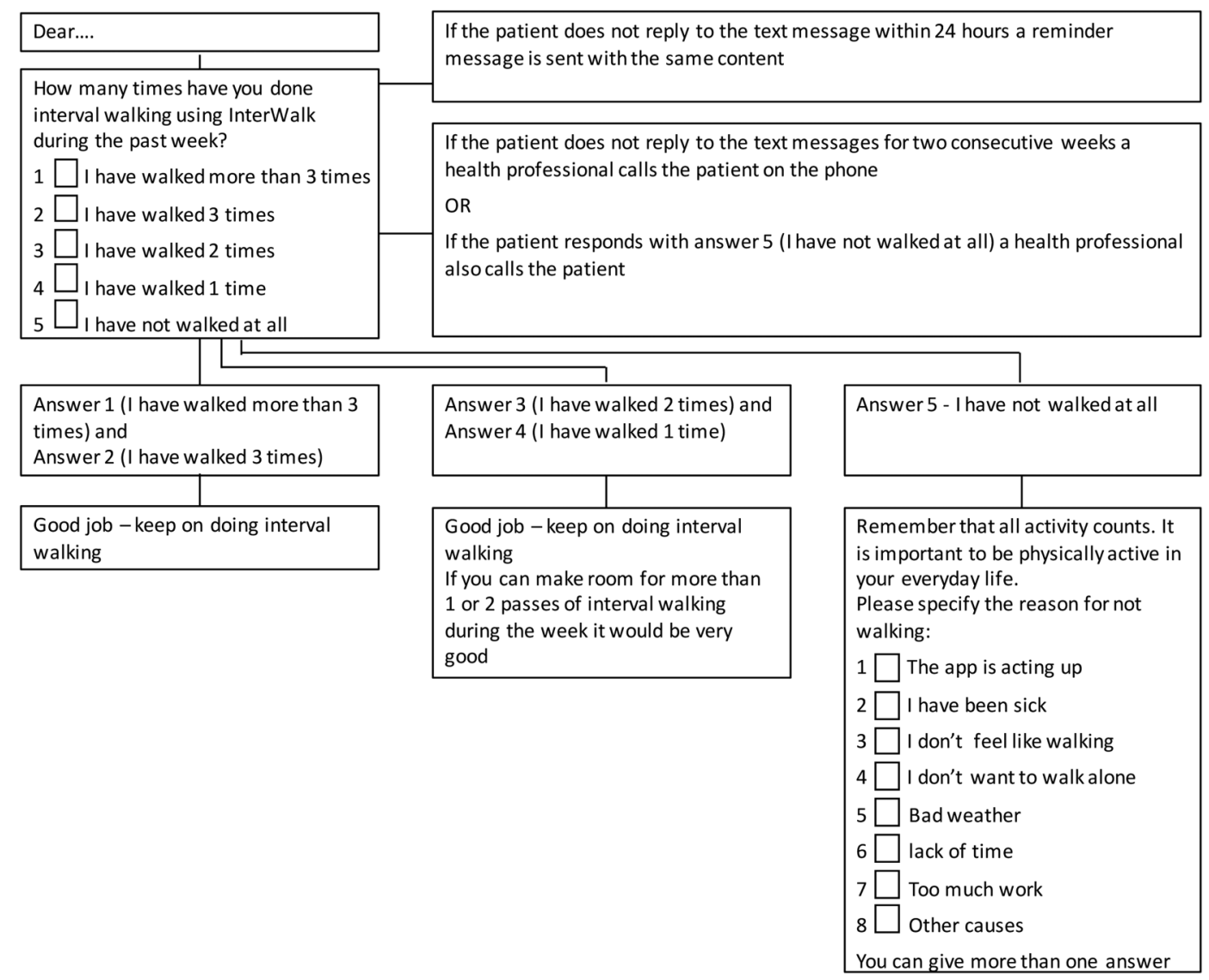

Figure 4 Short message service (SMS), send every Sunday to patients in the IWTsupport-group. IWT, Interval Walking Training. 
Short Message Service (SMS)

Standardized Walking test in the InterWalk application

\begin{tabular}{|l|}
\hline Dear.... \\
$\begin{array}{l}\text { It is time to adjust your walking test } \\
\text { The test is adjusted the same way you } \\
\text { initially did the test at the beginning of } \\
\text { the study at the health promotion } \\
\text { centre. }\end{array}$ \\
$\begin{array}{l}\text { Go to 'Settings' in the InterWalkapp } \\
\text { and then to 'personal customization' - } \\
\text { then do a new walking test }\end{array}$
\end{tabular}

Figure 5 Short message service (SMS), Standardised walking test in the InterWalk application, send every fourth week to patients in the IWTsupport-group. IWT, Interval Walking Training.

in IWTgroup have no contact with the health professionals until 52-weeks follow-up (see figure 1).

\section{Control intervention-standard care}

Patients in the control group receive the standard rehabilitation treatment offered to patients with T2D in Denmark in a multimodal setting including PA (phase I, figure 1). The duration of rehabilitation treatment differs between participating municipalities, but may not be shorter than 8 weeks during phase I. At the end of the standard care rehabilitation programme in the municipalities, there is no follow-up on the exercise intervention, which is the normal procedure in Danish municipalities ( phase II, figure 1). ${ }^{33}$

The participating municipalities offer group exercise twice a week at the health promotion centres with 6-15 patients participating depending on the population size in the participating municipality. The content of the group exercise period is based on clinical guidelines in Denmark. A typical session is structured with first a short warm-up session followed by a strength and cardio part and finished with a cool-down period with strengthening (see table 1). Furthermore, it is recommended that the patients have regularly consultation at the GP every third month.

\section{Additional rehabilitation care (cointerventions)}

All patients receiving rehabilitation at either a health promotion centre or at the hospital receives an offer of specific disease-related patient education, diet counselling and smoking cessation. ${ }^{33}$ It is optional whether the patient participates in one or all additional offers.

Disease-specific patient education includes a section focused on behaviour modification and selfmanagement and a specific part on diseases where the patients get knowledge regarding sequelae. Diet counselling regarding nutritional advice when living with T2D is handled by a dietitian. Smoking cessation is either group based or in individual process and is handled by smoking cessation counsellors.

\section{Trial end points and assessments}

Previous studies have established that PA influences glycaemic control ${ }^{34}$ and Karstoft $e t a l^{14}$ has shown that PA with IWT had effect on glycaemic control in patients with T2D. Moreover, medication and dietary interventions have also proven to improve glycaemic control and reduce disease-related complications. ${ }^{35-37}$ In the present trial, the primary outcome focus is on PA in relation to everyday life.

Data on the primary and secondary outcomes are obtained at baseline, postintervention (after 8-12 weeks) and 52 weeks after enrolment (table 3 ). To standardise all measurements between health professionals and participating centres, all health professionals use standardised protocols crafted for the trial regarding procedures and measurements. All data are entered directly in an online database (see section: Data management and quality control).

\section{Primary outcome}

The primary outcome is change in moderate-to-vigorous-intensity $\mathrm{PA}$ from baseline to 52-week follow-up.

\section{Secondary outcomes}

The key secondary outcome changes in motivation for sustaining a behavioural change with PA in everyday life at 52-week follow-up.

Other secondary outcomes of interest are changes in patients' fitness level measured as $\mathrm{VO}_{2}$-peak by the standardised walking test (part of the InterWalk application) and health-related quality of life. Strength in the lower extremities, adherence to intervention and impact of personality traits on PA in everyday life are also considered exploratory secondary outcomes. All outcomes are measured at baseline, after intervention and at 52-week follow-up (see figure 2).

\section{Data collection \\ Level of daily moderate-to-vigorous-intensity PA}

It is important to gain knowledge into PA in everyday life. To determine level of daily moderateto-vigorous-intensity PA, a hip-mounted PA monitor (Axivity AX3, Newcastle, UK) is worn for 7 consecutive days, at three time periods during the trial period; after baseline test, after the intervention period and again at 52-weeks follow-up. The monitor is fixed using adhesive tape (Leukomed T, BSN medical, Germany) on right side of the lumbar spine. Additionally, a monitor is fixed on the lateral side of the right thigh (Leukomed T, BSN medical, Germany) for supporting information about posture. ${ }^{24}$ The patients are instructed not to remove the PA monitor at any time during the three time periods. If de-attached, the participants are trained in fixing the 
Table 3 Summary of data collection and measures

\begin{tabular}{|c|c|c|c|c|}
\hline Measurements & Description & Baseline & 8-12 weeks & 52 weeks \\
\hline \multicolumn{5}{|l|}{ Demographic*†‡ } \\
\hline Age (median) & Obtained using self report & $x$ & - & - \\
\hline Women & & $x$ & - & - \\
\hline Marital status & & $x$ & - & - \\
\hline Diabetes duration & & $x$ & - & - \\
\hline Education level & & $x$ & - & - \\
\hline Height, weight, body mass index & Obtained using standard procedures & $x$ & $x$ & $x$ \\
\hline Medical history $† \ddagger$ & Obtained using medical records & & & \\
\hline History of heart disease & & $x$ & $x$ & $x$ \\
\hline Hypertension & & $x$ & $x$ & $x$ \\
\hline Kidney disease & & $x$ & $x$ & $x$ \\
\hline Chronic obstructive pulmonary disease (COPD) & Defined by $\mathrm{FEV}_{1}<70 \%$ & $\mathrm{x}$ & $\mathrm{x}$ & $\mathrm{x}$ \\
\hline \multicolumn{5}{|l|}{ 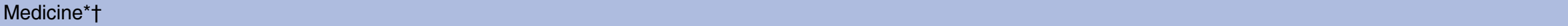 } \\
\hline Self-reported use of medication & & $x$ & $x$ & $x$ \\
\hline Register-data use of medicine & & $x$ & $x$ & $x$ \\
\hline \multicolumn{5}{|l|}{ Physical fitness* } \\
\hline Moderate-to-vigorous-intensity & $\begin{array}{l}\text { Accelerometer (Axivity AX3, Newcastle, UK), worn for } 7 \text { consecutive days, three } \\
\text { times }\end{array}$ & $x$ & $x$ & $\mathrm{x}$ \\
\hline Total physical activity (VO2-peak) & InterWalk-Walking test performed using an iPod touch or IPhone & $x$ & $\mathrm{x}$ & $x$ \\
\hline \multicolumn{5}{|l|}{ Strength* } \\
\hline Lower extremities & Sit-to-Stand & $x$ & $\mathrm{x}$ & $x$ \\
\hline \multicolumn{5}{|l|}{ Patient reported outcomes (PROs)* } \\
\hline Behavioural regulation in exercise & Obtained using BREQ-2, Behavioural Regulation in Exercise Questionnaire & $x$ & $x$ & $x$ \\
\hline Physical activity & Obtained using R-PAQ, Resent Physical Activity Questionnaire & $x$ & $x$ & $x$ \\
\hline Health-related quality of life & Obtained using SF-12, Short Form-Health-Related Quality of life Questionnaire & $x$ & $x$ & $x$ \\
\hline Automaticity of behaviour & The Self-Report Habit Index & & $x$ & $x$ \\
\hline Personality traits $†$ & $\begin{array}{l}\text { Obtained using NEO-FFI, Neuroticism, Extroversion and Openness-Five Factor } \\
\text { Inventory (Personality traits) }\end{array}$ & $\mathrm{x}$ & - & - \\
\hline Risk behaviourt & Obtained using SES, Sensation Seeking Scale (risk behaviour) & $x$ & $x$ & $x$ \\
\hline \multicolumn{5}{|l|}{ Adherence to interventions ${ }^{\star}$} \\
\hline Attendance registration & From baseline to $8-12$ weeks & $x$ & $x$ & $x$ \\
\hline Log in the InterWalk app & Measured by the electronic log in the InterWalk-app & $x$ & $x$ & $x$ \\
\hline \multicolumn{5}{|l|}{ Registry data assessment ${ }^{\star} \ddagger$} \\
\hline Mortality, cause of death & Obtained using medical records & - & $x$ & $\mathrm{x}$ \\
\hline Hospitalisation & & - & $x$ & $x$ \\
\hline Contract with general practitioner & & - & $x$ & $x$ \\
\hline
\end{tabular}

single-blinded randomised controlled trial.

†Article 2: Baseline characteristics and personality traits in patients with type 2 diabetes: descriptive study of all participants.

fArticle 3: Nay Sayers-characteristics of patients who does not want to be part of a physical activity study related to everyday life; all patients referred to the health promotion centre in the municipalities participating in the randomised controlled trial. 
monitors correctly and are instructed to note the incident. If the patients need help in fixing the monitors correctly, the health professionals at the health promotion centre are educated to help.

\section{PA diary}

To validate the PA level during the 7 days with PA monitors, we ask the patient to complete a 7-day diary to report number of work and sleep hours, bicycling and strength training sessions at the three time periods on a daily basis. The PA monitor and the diary are returned free-of-charge via mail. The patient is contacted by phone after within 1 week if the PA monitors are not returned as expected.

\section{Motivation for behaviour change with PA}

Changes in motivation for sustaining a behavioural change with PA in everyday life is measured with the Behavioural Regulation of Exercise Questionnaire (BREQ-2) which measures individual motivation for PA. The questionnaire consists of 19 questions in a 0-4 Likert format (Jensen et al Validation of the Behavioral Regulation for Exercise Questionnaire in Danish: measuring of motivation for exercise in people living with diabetes. 2016 (in preparation)). In addition to the questionnaire, patients are asked to answer four questions in a $0-4$ Likert format, regarding motivation for PA after being diagnosed with T2D.

\section{Health-Related Quality of Life}

The patient's Health-Related Quality of Life is very important to maintain after being diagnosed with T2D (Valentiner, et al Patients with T2D choose quality of life over health: a focus group investigation. 2016 (submitted)). To gain knowledge into the individual perception of quality of life on a physical and mental level we use the Short-Form Health Survey (SF-12). The questionnaire consists of 12 questions and uses a Likert scale of 1-3 for the physical function items; 1-5 for the bodily pain, social function and general health perceptions items; 1-6 for the vitality and mental health and a dichotomous scale of yes/no for the presence of role function limitations. The higher score, the higher level of health or functioning. ${ }^{38}$

\section{Maintenance of behaviour change with PA}

Automaticity of behaviour is essential when new habits become present in the everyday life. ${ }^{39}$ To measure if the patient has adopted new habits as a result of the intervention we ask the patient to answer four questions from The Self-Report Habit Index. The Index uses a 0-4 Likert format and the four questions to be answered measures automatic activation, frequency of behaviour and relevance to self-identity related to $\mathrm{PA}{ }^{40}$

\section{$\mathrm{VO}_{2}$-peak}

$\mathrm{VO}_{2}$-peak is measured by a validated and standardised walking test consisting of five stages incorporated in the
InterWalk app; (1) $30 \mathrm{~s}$ of standing still, (2) 2 min of slow walking, (3) 2 min of walking at moderate intensity, (4) 2 min walking at high intensity and (5) $1 \mathrm{~min}$ walking at highest intensity possible. The four paces are self-selected (see figures 3 and 4 ). ${ }^{24}$ The test is performed with an iPod placed in the pocket at the hip and the instructions are delivered from the app through earphones. To ensure the right walking intensity in the intervention period, the patients are asked to perform the test every fourth week. ${ }^{15}$

\section{Strength in the lower extremities}

The Sit-to-Stand test (30 s) measures the lower body strength and will be administered using a chair without arms. The patient is encouraged to complete as many full stands as possible within a $30 \mathrm{~s}$ time limit. ${ }^{41}$

\section{Adherence to the intervention}

Adherence is determined by an evaluation and quantification of the electronic log of data from the InterWalk app to the central database of all uploaded data.

\section{Self-rated PA}

The 'Recent Physical Activity Questionnaire' (RPAQ) has nine main questions, which covers four domains of PA: domestic life, work, recreation and transport. PA is estimated in METs (metabolic equivalent). In answering the questions in the questionnaire, the patient will answer in regards to the last 4 weeks with PA in the everyday life. ${ }^{42}$

\section{Personality traits}

The NEO-Five Factor Inventory (NEO-FFI) ${ }^{43}$ consists of 60 questions in a 0-4 Likert format constructed by selecting two items from each of the six facets characterising each of the five personality traits (Neuroticism, Extraversion, Openness, Agreeableness, Conscientiousness) assessed by NEO-PI-R. The Sensation Seeking Scale $(\mathrm{SES})^{44}$ is a 40 items self-administered questionnaire consisting of 40 questions designed to test the tendency towards varied, novel and intense sensations.

\section{Demographic, social economic and anthropometry measures}

Information on height, weight, waist and hip circumference is collected before inclusion through an electronic questionnaire at the first formal meeting at the health promotion centre and after the intervention period and again at 52-week follow-up. Waist circumference is measured at the point between the top of the iliac crest and the bottom of the costae regardless of the placement of the umbilicus. A Tanita stadiometer is used to measure height and an electronic weight to measure weight. Data on self-reported information together with relevant national registers will be used to obtain information regarding hospitalisation, comorbidities, medical history, use of medicine and mortality. 
Height measurement is conducted using a portable altimeter (Tanita stadiometer). The measurement is carried out without shoes and is repeated two times with each measure to nearest $0.1 \mathrm{~cm}$. If the two measures are dissimilar with $>0.5 \mathrm{~cm}$, then the measurement is repeated. Weight is measured in kilograms using an electronic weight. The measure is repeated two times with each measure to nearest $0.1 \mathrm{~cm}$. If the two measures are dissimilar with $>0.5 \mathrm{~kg}$, then the measurement is repeated a third time. The patient is weighed fully dressed and $1 \mathrm{~kg}$ is subtracted from each measure. Hip circumference is measured on the skin on the crest of the hipbone. The patient is asked to place a finger in the belly bottom and the measure is carried out with a tape measure $(2 \mathrm{~m})$ placed on the upper side of the patients' finger. If the two measures are dissimilar with $>0.5 \mathrm{~cm}$, then the measurement is repeated a third time.

\section{Safety criteria and adverse events}

Patients are informed that performing the InterWalk walking test may cause some degree of breathlessness as patients are expected to reach $80-85 \%$ of $\mathrm{VO}_{2}$-peak during the walking test. All other measurements are not associated with any known risk or discomfort. Injuries linked to the intervention are registered, if informed to the health professionals at the health promotion centres, during the trial period. In case of severe adverse events, the Scientific Ethical Committee of the Capital Region of Demark is informed.

\section{Sample size considerations}

The minimum difference of interest (MDI) is $10 \mathrm{~min}$ per day. Based on existing experimental evidence, we expect the SD of the change in moderate-and-vigorous PA from baseline to 12-month follow-up to be between 1.2 and 2.3 times the effect size. ${ }^{45} 46$ Therefore, SD twice the MDI (20 min of moderate-and-vigorous PA) per day was applied in the sample size calculations. After applying Bonferroni adjustment due to multiple comparisons in the three group trial a total of 190 participants are needed to obtain a statistical power (1- $\beta$ ) of $80 \%$ with an $\alpha$ of 0.017 (two-tailed). Allowing for an attrition rate of $30 \%, 272$ patients (91 in the control group and 181 in experimental group) are recruited. The setting enables recruitment until 15 December 2016. The sample size is truncated at 272 participants or the $\mathrm{N}$ reached at the end of recruitment period-whatever is reached first (see section 'Participants').

\section{Randomisation, sequence generation and allocation concealment}

After returning the PA monitors patients are randomised to either standard care or experimental group after baseline test (phase I). Allocation to either IWTgroup or IWTsupport-group on patients allocated to the experimental group is concealed until after the postintervention test (phase II). A health professional associated with the trial, telephones the patient and inform about random allocation after returning the PA monitors to either IWT-group or IWTsupport-group. The patients are stratified by gender to ensure an equal number across all groups. The allocation sequence is generated through a standardised computer programme by an independent researcher (RC). The allocation sequence is concealed until the trial arms are assigned. A flow of patients is depicted in figure 2.

\section{Blinding}

The scientific staff is blinded to patient allocation from baseline to the 52-week follow-up is completed for all patients. The health professionals at the promotion centre are not blinded to allocation to either standard care or experimental groups.

\section{Statistical methods}

All analyses will be conducted according to the intention-to-treat principle. Continuous end points will be analysed using repeated measures analysis of covariance in a mixed linear model. Categorical and dichotomous end points will be analysed with the use of logistic regression. The model will include with group, and sex as fixed effects, with the baseline value as a covariate.

All patients randomised to one treatment, will be analysed according to the treatment to which the patient was allocated, irrespective of whether they received this or some other treatment, or no treatment at all. The intention-to-treat (ITT) population will be handled all patients randomised to the three treatment arms, and the data set is equal to the 'all patients randomised set' (APRS). Missing values due to patients' absence from follow-ups or withdrawal from the study are to be expected in clinical trials. Several approaches are described for handling missing data in the ITT analysis, and among them 'baseline observation carried forward' (BOCF: ie, a null-responder imputation, where the level at baseline is also considered the last). A linear mixed model analysis includes all patients with a baseline assessment, and includes fixed and random factors. Thus, repeated linear mixed model method is chosen for the primary analyses in this trial (ie, no data imputation), whereas the BOCF imputation, as well as the 'Per Protocol' population will be applied for the purpose of sensitivity analysis. Also, we will explore whether potentially important covariates such as age, sex, disease duration, degree of overweight (using body mass index), and smoking status at enrolment/baseline could potentially confound the results from the primary analyses (ie, an extra prespecified sensitivity analysis). Exploratory analyses of the treatment effects will be performed on some the secondary outcomes.

\section{Data management and quality control}

All data are entered in an secure sockets layer (SSLsecured, online-based database developed by the Danish Stakeholder Help2Run (CVR: 34801088). The online- 
based database logistically handles the data gathered at baseline, after intervention in phase $\mathrm{I}$, and during follow-up (phase II) and after 52 weeks. Data on demography, measurements, tests and questionnaires are entered directly in the database available via a projectspecific homepage (http://www.runsafe.dk/mtd/login/ admin-signihttps://www.runsafe.dk/mtd/login/adminsignin). A protected back-end system (https://www. runsafe. $\mathrm{dk} / \mathrm{mtd} / \mathrm{admin}$ ) is used to logistically handle information on demography, measurements, tests and questionnaires on each participant in the trial (for information on IWT using the InterWalk app see 'Interval Walking Training using The InterWalk Application'. The system was approved in 2014 after an inspection by the Danish Data Protection Agency. Access to personalised data from the InterWalk participants is only possible using a personalised username and password. Only specified researchers are allowed to administrate the back-end system and each researcher and project member with access to the back end has a unique username and personal password for the back-end system. All activities, including changing data and downloading data, in the back-end system are logged in accordance with the rules and regulations from the Danish Data Protection Agency. A unique subject identification (ID) number will be subscribed to all patients to anonymise data. The identification key (ID to personal information) will be encrypted and stored securely and separately from the unique ID number on a secure database.

\section{Ethics and dissemination}

The InterWalk trial contributes with important knowledge on different treatment approaches in the rehabilitation of patients with T2D. PA and exercise have beneficial effect on patients with T2D when the interventions are conducted in well-designed efficacy studies. $^{2}{ }^{4}{ }^{6}$ However, little is known on whether this effect also is present in effectiveness studies or 'realworld setting' studies. These studies are of great importance and relevance regarding evidence-based medicine highlighting the importance of the InterWalk trial.

The InterWalk trial has a pragmatic design that allows for different intervention periods in the participating municipalities. Furthermore, the interventions are conducted directly in the clinical setting giving a more reliable frame of the experienced challenges in a population with T2D. We believe that the implementation of the study results will have a smoother implementation phase, as the health professionals already are familiar with the preceding's.

The trial gives valuable insight into the impact of different motivational and supportive tools as well as knowledge about individual motivation and the impact on sustaining a newly acquired behaviour change regarding PA in everyday life.

IWT is expected to be a suitable and effective way of providing moderate-to-vigorous PA for at large group of patients with T2D. IWT can be carried out at anytime and anywhere and is thus integrated and tailored to fit individual everyday life. The overall objective is to promote lifelong PA for the individual and we believe that experiences from the present trial can help when planning future interventions with IWT to patients with various lifestyle diseases, for example, coronary heart diseases.

It is a major advantage that the trial is designed as a randomised controlled trial in a 'real-world setting' with a study population representing patients with T2D referred to rehabilitation. Furthermore, data and results are collected consecutively and directly in the clinical setting in which the interventions are to be used after the trial. Together with the very limited number of inclusion and exclusion criteria and a stratification of patients on gender secures external validity of the trial and thus will add important information to the current body of evidence on T2D treatment.

All data from eHealth solutions as the InterWalk app are electronically monitored. The patient is not dependent on opening hours at the health promotion centre as the InterWalk is either installed on an iPhone or an iPod. This gives a unique possibility for the patient to be physically active whenever it fits in their everyday life.

We acknowledge that PA only has effect when adherence is high, which is why we first compare standard care with interval walking for a shorter period (phase I) followed by a reallocation of patients in the experimental group to either interval walking with or without motivational support (phase II). All groups have a 52-week follow-up. The trial design enables us to investigate if patients are able to maintain a newly acquired behaviour change with $\mathrm{PA}$ in everyday life with or without motivational support for a longer period.

All personalised data and measures are stored on a central online-based database developed to logistically handle all data collected at baseline and during follow-up. The back-end system (https://www.runsafe. $\mathrm{dk} / \mathrm{mtd} / \mathrm{admin} /$ ) secures all information from each patient. The construction of the database with personalised usernames, passwords and specific permissions to all health professionals and administering permissions to scientific staff in the back end secures all data. Furthermore, all activities are logged.

All outcomes measures are assessed blinded to allocation at baseline and the primary outcome is assessed blinded to intervention in the experimental group after the intervention period. All measures are conducted by the health professionals at the health promotion centres and are blinded for allocation after intervention in the experimental group with IWT until after return of the PA monitors by the patient. All statistical analyses are blinded to researchers, reducing the risk of detection and interpretation bias. ${ }^{47-49}$ All secondary outcomes, but $\mathrm{VO}_{2}$-peak and the Sit-to-Stand Test are self-reported and by nature likely to be biased. The patients answer 
questionnaires independently of the health professionals at the health promotion centres.

The secondary outcomes of motivation for PA and behaviour change are of highly importance as knowledge concerning motivational support and maintenance of effect (new habits with PA) to the best of our knowledge is lacking in patients with T2D. This knowledge will help health professionals to better tailor the treatment and herby potentially achieve a higher adherence to the programme following the supervised intervention.

The limitations in this trial are similar to those of other exercise trials where physical testing and time-of-day and day-to-day variation constitute a challenge. We have standardised all testing protocols for all procedures to reduce the variation and run a routine programme of ongoing calibration and training of the health professionals.

The trial is conducted in accordance with the Helsinki Declaration II. ${ }^{50}$ All eligible patients receive written and oral informed consent prior to inclusion before any additional trial procedures. Data on all screened patients are registered to report characteristics of ineligible patients and written informed consent is also obtained from these patients. The patients can withdraw from the trial at all times during the trial period. This has no consequence for any other future treatment. If patients discontinue the trial intervention, standard care is offered for the rest of the intervention period. All results from the trial, negative, positive or inconclusive results, are disseminated in international peer-reviewed scientific papers and at national and international conferences. We follow the guidelines from the International Committee of Medical Journal Editors when authorship is determined. The results will be reported when all long-term data become accessible.

Furthermore, all results are shared with the participating municipalities and participating health professionals and are used to develop future intervention and implementation strategies in the Danish municipalities.

\section{Trial status}

The inclusion period started in January 2015 and is scheduled to finish in December 2017 with a 52-week follow-up. To ensure enrolment of patients in the trial, we are open for collaborations with new municipalities during the trial period. So far, the trial has resulted in collaboration with rural and urban municipalities in Denmark. The inclusion rate is carefully monitored every week by the research team. In November 2016, 216 patients had been enrolled in the trial.

\footnotetext{
Author affiliations

${ }^{1}$ CopenRehab, Department of Public Health, Section of Social Medicine, Henrik Pontoppidans Vej 6, University of Copenhagen, Copenhagen, Denmark ${ }^{2}$ Department of Infectious Diseases, Center for Physical Activity Research, Rigshospitalet, University of Copenhagen, Copenhagen, Denmark

${ }^{3}$ The Danish Diabetes Academy, Odense University Hospital, Odense, Denmark ${ }^{4}$ Department of Infectious Diseases, Centre of Inflammation and Metabolism, Rigshospitalet, University of Copenhagen, Copenhagen, Denmark
}

${ }^{5}$ Department of Endocrinology (Diabetes and Metabolism), Rigshospitalet, University of Copenhagen, Copenhagen, Denmark

${ }^{6}$ Musculoskeletal Statistics Unit, The Parker Institute, Bispebjerg and

Frederiksberg Hospital, Copenhagen, Denmark

${ }^{7}$ Department of Endocrinology, Odense University Hospital, Odense, Denmark ${ }^{8}$ OPEN, Odense Patient data Explorative Network, Odense University Hospital, Odense, Denmark

Acknowledgements The authors thank the health professionals in the Municipalities of Copenhagen and Guldborgsund and Bornholm Hospital in Denmark for helping recruit, test and train with all patients in the study.

Collaborators The Municipality of Copenhagen is a collaborator in the trial. The Municipality of Guldborgsund is a collaborator in the trial. Bornholm Hospital is a collaborator in the trial. Assigned Health professionals working in the Municipality of Copenhagen, The Municipality of Guldborgsund and at Bornholm Hospital all contributed to the trial, by recruiting, testing and training with participating patients in the trial.

Contributors LSV, MR-L and HL drafted the manuscript. All authors made substantial contributions to conception and design, and revised the manuscripts critically for important intellectual content. All authors have given their approval for the manuscript to be published.

Funding The work has been supported by two grants by the Tryg Foundation, Denmark. The Centre for Physical Activity Research (CFAS) is supported by a grant from TrygFonden. The study was further supported by grants from. The Augustinus Foundation and two foundations in the Capital Region of Denmark - the Foundation for Prevention and the Foundation for Intersectorial

Research Projects have also supported the work. LSV is supported by a grant from the Municipality of Copenhagen, Denmark and The Danish

Physiotherapist Foundation. MR-L is supported by a postdoctoral grant from the Danish Diabetes Academy supported by the Novo Nordisk Foundation and The Vissing Foundation. CIM/CFAS is a member of DD2-the Danish Center for Strategic Research in Type 2 Diabetes (the Danish Council for Strategic Research, grant numbers 09-067009 and 09-075724). RC is supported by grants from The Oak Foundation. $\mathrm{HL}$ is supported by a professor grant from the Municipality of Copenhagen, Denmark.

Competing interests None declared.

Ethics approval The local regional Research Ethics Committee in Denmark (H-1-2014-074) and the Danish Data Protection Agency (j.nr. 2014-54-0897) has approved the trial.

Provenance and peer review Not commissioned; externally peer reviewed.

Data sharing statement Remaining study material not designated to planned articles (specified in the protocol article) is available by contacting the corresponding author (LSV). If relevant, a proposal can be made to the InterWalk steering committee. If approved, data are available.

Open Access This is an Open Access article distributed in accordance with the Creative Commons Attribution Non Commercial (CC BY-NC 4.0) license, which permits others to distribute, remix, adapt, build upon this work noncommercially, and license their derivative works on different terms, provided the original work is properly cited and the use is non-commercial. See: http:// creativecommons.org/licenses/by-nc/4.0/

\section{REFERENCES}

1. Colberg SR, Sigal RJ, Fernhall B, et al. Exercise and type 2 diabetes: the American College of Sports Medicine and the American Diabetes Association: joint position statement executive summary. Diabetes Care 2010;33:2692-6.

2. Plotnikoff RC, Costigan SA, Karunamuni ND, et al. Community-based physical activity interventions for treatment of type 2 diabetes: a systematic review with meta-analysis. Front Endocrinol (Lausanne) 2013:4:3.

3. Umpierre D, Kramer CK, Leita CB, et al. Clinician's Corner physical activity advice only or structured with $\mathrm{HbA} 1 \mathrm{c}$ levels in type 2 diabetes. JAMA 2013;305:190-9.

4. Nicolucci A, Balducci S, Cardelli P, et al. Relationship of exercise volume to improvements of quality of life with supervised exercise training in patients with type 2 diabetes in a randomised controlled 
trial: the Italian Diabetes and Exercise Study (IDES). Diabetologia 2012:55:579-88.

5. Vadstrup ES, Frølich A, Perrild $\mathrm{H}$, et al. Health-related quality of life and self-related health in patients with type 2 diabetes: effects of group-based rehabilitation versus individual counselling. Health Qual Life Outcomes 2011;9:110.

6. Avery L, Flynn D, Van Wersch A, et al. Changing physical activity behavior in type 2 diabetes: a systematic review and meta-analysis of behavioral interventions. Diabetes Care 2012;35:2681-9.

7. Shaw JE, Sicree RA, Zimmet PZ. Global estimates of the prevalence of diabetes for 2010 and 2030. Diabetes Res Clin Pract 2010;87:4-14.

8. Whiting DR, Guariguata L, Weil C, et al. IDF Diabetes Atlas: global estimates of the prevalence of diabetes for 2011 and 2030. Diabetes Res Clin Pract 2011;94:311-21.

9. Cochrane Collaboration. Special Collection: health of Indigenous peoples: diabetes. 2015. http://www.cochranelibrary.com/app/ content/special-collections/article/?doi=10.1002/(ISSN)14651858 (CAT)Freeaccesstoreviews(VI)indigenoushealthdiabetes

10. Policy EHealth, Europa.eu. https://ec.europa.eu/health/ehealth/ policy_en

11. Di Loreto C, Fanelli C, Lucidi $P$, et al. Make your diabetic patients walk: long-term impact of different amounts of physical activity on type 2 diabetes. Diabetes Care 2005;28:1295-302.

12. Eakin EG, Reeves MM, Lawler SP, et al. The Logan Healthy Living Program: a cluster randomized trial of a telephone-delivered physical activity and dietary behavior intervention for primary care patients with type 2 diabetes or hypertension from a socially disadvantaged community - rationale, design and recruitment. Contemp Clin Trials 2008;29:439-54.

13. Tatara N, Årsand E, Bratteteig T, et al. Usage and perceptions of a mobile self-management application for people with type 2 diabetes: qualitative study of a five-month trial. Stud Health Technol Inform 2013;192:127-31.

14. Karstoft $\mathrm{K}$, Winding $\mathrm{K}$, Knudsen $\mathrm{SH}$, et al. The effects of free-living interval-walking training on glycemic control, body composition, and physical fitness in type 2 diabetic patients: a randomized, controlled trial. Diabetes Care 2013;36:228-36.

15. Ried-larsen M, Thomsen RW, Berencsi K, et al. Implementation of interval walking training in patients with type 2 diabetes in Denmark: rationale, design, and baseline characteristics. Clincal Epidemiol 2016;8:201-9.

16. Saida T, Langberg H. Self-Reported adherence to physical activity following physical activity referral scheme \& objective measured physical activity in self-referred subgroup: a pragmatic 12 month follow-up study. 2017 (in press).

17. Ogden J, Hills L. Understanding sustained behavior change: the role of life crises and the process of reinvention. Health (London) 2008;12:419-37.

18. Chan AW, Tetzlaff JM, Gøtzsche PC, et al. SPIRIT 2013 explanation and elaboration: guidance for protocols of clinical trials. $B M J$ 2013;346:e7586.

19. Schulz KF, Altman DG, Moher D, CONSORT Group. CONSORT 2010 statement: updated guidelines for reporting parallel group randomized trials. Ann Intern Med 2010;152:726-32.

20. Thomsen RW, Nielsen JS, Ulrichsen SP, et al. The Danish Centre for Strategic research in type 2 diabetes (DD2) study: collection of baseline data from the first 580 patients. Clin Epidemiol 2012;4:43-8.

21. Thygesen LC, Daasnes C, Thaulow I, et al. Introduction to Danish (nationwide) registers on health and social issues: structure, access, legislation, and archiving. Scand J Public Health 2011;39:12-16.

22. Bodenheimer T, Wagner EH, Grumbach K. Improving primary care for patient with chronic illness: the chonic care model, Part 2. JAMA 2002;288:1909-14.

23. Pedersen BK, Saltin B. Evidence for prescribing exercise as therapy in chronic disease. Scand J Med Sci Sports 2006;16(Suppl 1):3-63.

24. Brinkløv CF, Thorsen IK, Karstoft $\mathrm{K}$, et al. Criterion validity and reliability of a smartphone delivered sub-maximal fitness test for people with type 2 diabetes. BMC Sports Sci Med Rehabil 2016;8:31.

25. Locke EA. Toward a theory of task motivation and incentives. Organ Behav Hum Perform 1968;3:157-89.

26. Miller WR, Rollnick S. Motivational interviewing: preparing people for change. 2nd edn. New York, NY: Guilford Press, 2002.
27. Chen SM, Creedy D, Lin HS, et al. Effects of motivational interviewing intervention on self-management, psychological and glycemic outcomes in type 2 diabetes: a randomized controlled trial. Int J Nurs Stud 2012;49:637-44.

28. Locke EA, Latham GP. New directions in goal-setting theory. SAGE 2006;15:265-8.

29. Fjeldsoe BS, Marshall AL, Miller YD. Behavior change interventions delivered by mobile telephone short-message service. Am J Prev Med 2009;36:165-73.

30. Ryan RM, Deci EL. Intrinsic and extrinsic motivations: classic definitions and new directions. Contemp Educ Psychol 2000;25:54-67.

31. Shaya TF, Chirikov VV, Howard D, et al. Effect of social networks intervention in type 2 diabetes: a partial randomised study. J Epidemiol Community Heal 2014;68:326-32.

32. Paul G, Keogh K, D'Eath M, et al. Implementing a peer-support intervention for people with type 2 diabetes: a qualitative study. Fam Pract 2013;30:593-603.

33. Drivsholm TB, Snorgaard O. Organisation of treatment and follow up of patients with type 2 diabetes. J Danish Med Assoc 2012;174:2159-62.

34. Snowling NJ, Hopkins WG. Effects of different modes of exercise training on glucose control and risk factors for complications in type 2 diabetic patients: a meta-analysis. Diabetes Care 2006;29:2518-27.

35. Ajala O, English P, Pinkney J. Systematic review and meta-analysis of different dietary approaches to the management of type 2 diabetes. Am J Clin Nutr 2013;97:505-16.

36. Evert $A B$, Boucher JL, Cypress M, et al. Nutrition therapy recommendations for the management of adults with diabetes. Diabetes Care 2013;36:3821-42

37. Palmer SC, Mavridis D, Nicolucci A, et al. Comparison of clinical outcomes and adverse events associated with glucose-lowering drugs in patients with type 2 diabetes: a meta-analysis. JAMA 2016;316:313-24.

38. Ware J Jr, Kosinski M, Keller S. A 12-Item Short-Form Health Survey: construction of scales and preliminary tests of reliability and validity. Med Care 1996;34:220-33.

39. Gardner B. A review and analysis of the use of "habit" in understanding, predicting and influencing health-related behaviour. Health Psychol Rev 2015;9:277-95.

40. Gardner B, Abraham C, Lally P, et al. Towards parsimony in habit measurement: testing the convergent and predictive validity of an automaticity subscale of the Self-Report Habit Index. Int J Behav Nutr Phys Act 2012;9:102.

41. Jones CJ, Rikli RE, Beam WC. A 30-s chair-stand test as a measure of lower body strength in community-residing older adults. Res Q Exerc Sport 1999;70:113-19.

42. Golubic R, May AM, Benjaminsen Borch K, et al. Validity of electronically administered recent physical activity questionnaire (RPAQ) in ten European countries. PLoS One 2014;9:e92829.

43. Mortensen EL, Flensborg-Madsen T, Molbo D, et al. Personality in late midlife: associations with demographic factors and cognitive ability. J Aging Health 2014;26:21-36.

44. Zuckerman M, Kolin EA, Price L, et al. Development of a sensation-seeking scale. 1964;28:477-82.

45. Kirk A, Mutrie N, Maclntyre P, et al. Effects of a 12-month physical activity counselling intervention on glycaemic control and on the status of cardiovascular risk factors in people with type 2 diabetes. Diabetologia 2004;47:821-32.

46. De Greef K, Deforche B, Tudor-Locke C, et al. A cognitive-behavioural pedometer-based group intervention on physical activity and sedentary behaviour in individuals with type 2 diabetes. Health Educ Res 2010;25:724-36.

47. Savović J, Jones HE, Altman DG, et al. Influence of reported study design characteristics on intervention effect estimates from randomized, controlled trials. Ann Intern Med 2012;157:429-38.

48. Wood L, Egger M, Gluud LL, et al. Empirical evidence of bias in treatment effect estimates in controlled trials with different interventions and outcomes: meta-epidemiological study. $\mathrm{Bm}$ 2008:336:601-5.

49. Gluud LL. Bias in clinical intervention research. Am J Epidemiol 2006;163:493-501.

50. World Medical Association. World Medical Association Declaration of Helsinki: ethical principles for medical research involving human subjects. JAMA 2013;310;2191-94. 